\title{
Correction to: Similar spatial patterns of soil quality indicators in three poplar-based silvo-arable alley cropping systems in Germany
}

\author{
René Beuschel ${ }^{1} \cdot$ Hans-Peter Piepho ${ }^{2} \cdot$ Rainer Georg Joergensen ${ }^{1} \cdot$ Christine Wachendorf $^{1}$
}

Published online: 29 October 2018

(C) Springer-Verlag GmbH Germany, part of Springer Nature 2018

\section{Correction to: Biology and Fertility of Soils \\ https://doi.org/10.1007/s00374-018-1324-3}

Table 2 of the original version of this article unfortunately contains the superscript letters "\#" and "\$" within columns instead of the stastistical letters "a" and "b". Superscript letters "\#" and "\$" within columns were changed to "a" and "b", respectively. The correct Table 2 is as follows:

The online version of the original article can be found at https://doi.org/ $10.1007 / \mathrm{s} 00374-018-1324-3$

René Beuschel

r.beuschel@uni-kassel.de

1 Department of Soil Biology and Plant Nutrition, University of Kassel, Nordbahnhofstraße 1a, 37213 Witzenhausen, Germany

2 Biostatistics Unit, Institute of Crop Science, University of Hohenheim, Fruwirthstraße 23, 70593 Stuttgart, Germany 
Table 2 Mean values of soil organic C (SOC), soil microbial biomass C $(\mathrm{MBC})$ and $\mathrm{N}(\mathrm{MBN})$, ergosterol and the ratios SOC to total $\mathrm{N}(\mathrm{TN})$, ergosterol to fungal glucosamine $(\mathrm{GlcN})$ and fungal $\mathrm{C}$ to bacterial $\mathrm{C}$ at different distances from the tree rows at two soil depths combined for three alley cropping systems. Clay content and $\mathrm{pH}$ were used as covariates. Mean values and probability values based on the GLIMMIX procedure

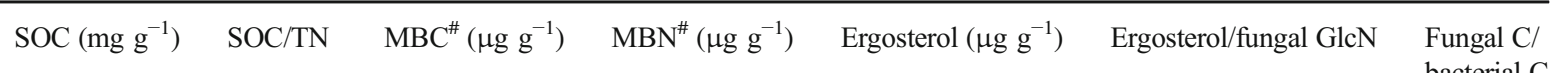

\begin{tabular}{|c|c|c|c|c|c|c|c|}
\hline \multicolumn{8}{|l|}{$0-5 \mathrm{~cm}$} \\
\hline Tree row & $21.1^{\mathrm{a} * *}$ & $11.3^{\mathrm{a} * * *}$ & $541(6.3)^{\mathrm{a}} * * *$ & $85(4.4)^{\mathrm{a} * *}$ & $4.95^{\mathrm{a}} * * *$ & $5.84^{\mathrm{a} *}$ & $3.10^{\mathrm{a}} *$ \\
\hline $1 \mathrm{~m}$ & $18.4^{\mathrm{b}}$ & $10.7^{\mathrm{b}}$ & $357(5.9)^{\mathrm{b}} *$ & $65(4.2)^{\mathrm{b}}$ & $2.50^{\mathrm{b}}$ & $3.39^{\mathrm{b}}$ & $2.59^{\mathrm{b}}$ \\
\hline $7 \mathrm{~m}$ & $17.4^{\mathrm{b}}$ & $10.5^{\mathrm{b}}$ & $299(5.7)^{\mathrm{b} *}$ & $52(3.9)^{\mathrm{b}}$ & $2.12^{\mathrm{b}}$ & $2.78^{\mathrm{b}}$ & $2.61^{\mathrm{b}}$ \\
\hline $24 \mathrm{~m}$ & $17.7^{\mathrm{b}}$ & $10.6^{\mathrm{b}}$ & $340(5.8)^{\mathrm{b} *}$ & $59(4.1)^{\mathrm{b}}$ & $2.48^{\mathrm{b}}$ & $3.48^{\mathrm{b}}$ & $2.53^{\mathrm{b}}$ \\
\hline SED & 0.6 & 0.2 & $0.08^{\$}$ & $0.10^{\$}$ & 0.28 & 0.58 & 0.10 \\
\hline \multicolumn{8}{|c|}{ Probability values } \\
\hline Clay & 0.008 & ns & ns & $<0.001$ & ns & ns & ns \\
\hline $\mathrm{pH}$ & ns & ns & ns & ns & ns & 0.01 & 0.04 \\
\hline \multicolumn{8}{|l|}{$5-20 \mathrm{~cm}$} \\
\hline Tree row & $17.0^{\mathrm{a}}$ & $10.5^{\mathrm{a}}$ & $282(5.6)^{\mathrm{a}}$ & $41(3.7)^{\mathrm{a}}$ & $1.86^{\mathrm{a}}$ & $2.50^{\mathrm{a}}$ & $2.92^{\mathrm{a}}$ \\
\hline $1 \mathrm{~m}$ & $17.8^{\mathrm{a}}$ & $10.7^{\mathrm{a}}$ & $282(5.6)^{\mathrm{a}}$ & $47(3.8)^{\mathrm{a}}$ & $1.85^{\mathrm{a}}$ & $1.93^{\mathrm{a}}$ & $2.59^{\mathrm{a}}$ \\
\hline $7 \mathrm{~m}$ & $17.8^{\mathrm{a}}$ & $10.6^{\mathrm{a}}$ & $268(5.6)^{\mathrm{a}}$ & $46(3.8)^{\mathrm{a}}$ & $1.53^{\mathrm{a}}$ & $1.82^{\mathrm{a}}$ & $2.49^{\mathrm{a}}$ \\
\hline $24 \mathrm{~m}$ & $17.2^{\mathrm{a}}$ & $10.5^{\mathrm{a}}$ & $264(5.6)^{\mathrm{a}}$ & $44(3.8)^{\mathrm{a}}$ & $1.73^{\mathrm{a}}$ & $2.15^{\mathrm{a}}$ & $2.52^{\mathrm{a}}$ \\
\hline SED & 0.5 & 0.2 & $0.09^{\$}$ & $0.16^{\$}$ & 0.29 & 0.49 & 0.16 \\
\hline \multicolumn{8}{|c|}{ Probability values } \\
\hline Clay & 0.01 & $\mathrm{~ns}$ & ns & $<0.001$ & $<0.001$ & ns & ns \\
\hline $\mathrm{pH}$ & ns & $<0.001$ & ns & 0.005 & ns & 0.03 & 0.02 \\
\hline
\end{tabular}

Means with no letter in common within a column indicate significant differences between distances of each soil depth (Tukey-Kramer, $P<0.05), n=12$; asterisks show significant differences between soil depths of each distance (probability values were Bonferroni-Holm corrected for multiple testing; $* * *$ $P<0.001, * * P<0.01, * P<0.05)$; SED: mean standard error of differences between distances of each soil depth; ns: not significant; if covariates were significant, covariate-adjusted means are presented; all two- and threefold interactions were insignificant. Ergosterol to fungal GlcN presented in $\mu \mathrm{g}$ $\mathrm{mg}^{-1}$.

\# Back-transformed data from the logarithmic scale are presented as medians and data on the logarithmic scale in brackets

$\$$ Mean standard error of differences between distances on the logarithmic scale 